\title{
Restricciones al crecimiento de la productividad en el Ecuador: Brecha externa y fiscal (2002-2017)
}

\author{
Nicolás Acosta-González'; Erik Camelos² \\ Fecha de recepción: 23 de Agosto 2018 \\ Fecha de aceptación: 21 de Septiembre 2018
}

\section{Resumen}

En el siguiente artículo se presentan teóricamente los equilibrios fundamentales de una economía, se encuentra que en el Ecuador los dominantes son el externo y fiscal. Por lo que, la vulnerabilidad del sector externo ante factores exógenos afecta a la brecha fiscal y a la actividad productiva en conjunto, dado que el Ecuador se presenta como una 'State led economy'. La solución común ha sido la contratación de deuda pública que canalice la brecha fiscal y genere ajuste con crecimiento. No obstante, la inversión pública no evoluciona positivamente con el crecimiento del stock de deuda, esto debilita la productividad de la economía y consecuentemente agrava la sostenibilidad de la deuda.

Palabras clave: Brecha externa, déficit fiscal, productividad, deuda pública, sostenibilidad.

\section{Abstract}

The following paper attempts to show the fundamental balances of an economy, it is found that, in Ecuador the dominant are the external and fiscal balances. So, external balance vulnerability in the face of exogenous shocks, ends affecting the fiscal balance and productive activity in a whole, given that Ecuador is presented as a 'State led economy'. The common solution has been the increment of public debt that channel the fiscal gap and generate adjustment with growth. Nevertheless, public investment does not oscillate positively as the debt stock increment does, this weakens the economy's productivity and consequently aggravates the debt sustainability.

Keywords: External gap, fiscal gap, productivity, public debt, sustainability. Introducción

La economía del Ecuador después del proceso de dolarización de inicios del siglo XXI atravesó por una breve recuperación con la ayuda de flujos positivos por un incremento en el precio del barril de petróleo que aportaron a la consolidación de niveles adecuados en los indicadores macroeconómicos.

Durante el siglo XXI, dos han sido los manejos de política económica que han trascendido el contexto macro. El elemento diferenciador entre el periodo 2000-2006 y 2007-2017 fue el alto nivel de desahorro público evidenciado en el crecimiento de la inversión pública en el segundo periodo que, posicionó al Estado ecuatoriano como motor del crecimiento económico.

Sin embargo, los relativamente altos ingresos externos reflejados en la holgura de la brecha externa que permitieron maximizar el desahorro público se relacionan de manera directa con el auge del precio del barril de petróleo, lo que vulneró la brecha fiscal (pública) cuando el precio del crudo cayó abruptamente en 2014, ocasionando iliquidez en el sector público que se vio obligado a contraer mayores volúmenes de deuda para mantener los niveles de inversión y gasto que han nutrido mayormente a la actividad productiva de la economía ${ }^{3}$.

En este sentido, la contratación de deuda pública se ha posicionado como una estrategia de corto plazo capaz de canalizar la golpeada brecha fiscal y empujar a un ajuste con crecimiento. No obstante, el stock de deuda pública ha aumentado desde 2009 y se disparó desde 2014 superando al stock promedio del primer periodo, alcanzando al cierre de 2017 un 45\% del PIB, lo que reaviva la discusión sobre la sostenibilidad de la deuda.
Cabe mencionar que, a mediados de la última década del siglo anterior León, Celi y Carvajal llevaron a cabo el cálculo de las tres brechas (pública, privada y externa) para el Ecuador de 1965 a 1993, sentando un precedente de la aplicación del modelo de brechas para su reflexión en la economía ecuatoriana.

En base a lo expuesto anteriormente, el presente artículo busca exponer la relación existente entre la brecha externa y fiscal, teniendo en cuenta que, debido a la endogeneidad monetaria ${ }^{4}$, resultado de la adopción de la dolarización, la economía ecuatoriana enfrenta una restricción externa derivada de la demanda mundial que es exógena. En este artículo se busca exponer la manera en que la restricción externa determina la brecha fiscal y por ende el rumbo de la actividad económica en una 'state led economy' como la del Ecuador.

\section{Metodología}

Para el presente artículo se ha considerado un enfoque inductivo ya que como se verá posteriormente, se parte de la teoría y, un método cuantitativo debido a que los planteamientos e hipótesis a lo largo de la investigación se sustentan en evidencia empírica obtenida de las fuentes oficiales de información como el Banco Central del Ecuador (BCE) y Ministerio de Finanzas (MEF).

En función los datos requeridos para el estudio, se procedió a desarrollar un análisis correlacional comparativo entre variables para dos periodos, 2000-2006 y, 2007-2017 con el fin de evidenciar empíricamente el comportamiento de la economía ecuatoriana y contrastarlo con una perspectiva teórica. Se utiizan datos a precios constantes del año 2010.

\section{Resultados}

Con la finalidad de llevar a cabo un estudio profundo de los equilibrios fundamentales de la economía, el análisis se basa en el modelo de brechas de Bacha $(1990)^{5}$. Estas brechas representan los equilibrios fundamentales que permiten a una economía desarrollar de manera adecuada su actividad productiva generando crecimiento económico en base al nivel de inversión.

Se contemplan las brechas o ahorros de tres sectores, sector externo, privado y público (fiscal). La estructuración de las identidades que hacen posible determinar cada brecha parte de principios contables de la lógica 'ingresos-egresos'. En este sentido, se procederá a desarrollar las brechas en base a las identidades macroeconómicas generalmente aceptadas, posterior a esto se pasará a estructurarlas en función de las cuentas nacionales.

Se parte de la identidad que determina que el ingreso es igual al consumo más la inversión:

$$
Y=C+l(1)
$$

Dado que en la lógica económica el ahorro es igual a la inversión, se igualan el consumo más la inversión y el consumo más el ahorro, de modo que se anula el consumo y se obtiene la identidad económica de que el ahorro es igual a la inversión.

$$
\mathrm{C}+\mathrm{l}=\mathrm{C}+\mathrm{S}
$$$$
S=/(2)
$$

Se procede a enunciar la ecuación de la demanda agregada keynesiana.

$$
Y=C+l+G+(X-M)(3)
$$

Por motivos de procedimiento matemático se asume que la relación $(X-M)=0$

'Master of Arts in Economics por Georgetown University. Director del Reporte de Consistencia Macroeconómica-RCM. Profesor de la Facultad de Economía de la Pontificia Universidad Católica del Ecuador. Quito- Ecuador. E-Mail: hnacosta@puce.edu.ec

${ }^{2}$ Reporte de Consistencia Macroeconómica (RCM). Pontificia Universidad Católica del Ecuador. Quito. Ecuador. E-mail: ecamelos039@puce.edu.ec

${ }^{3}$ Considerando que en Ecuador el negocio petrolero es estatal y representa la mayoría de las exportaciones.

${ }^{4}$ Cuando la cantidad de divisas depende únicamente de la producción nacional.

${ }^{5}$ Referencias de modelos de brechas en Chenery \& Bruno (1962), Chenery \& Strout (1966), Bacha (1984) y McKinnon (1964). 
$Y=C+1$

Generalizando la identidad $\mathrm{S}=\mathrm{I}$ se puede desagregar entre componentes privados y de gobierno.

$$
\begin{aligned}
& S=l=l p+l g=S p+S g(4) \\
& Y=C p+C g+S p+S g \text { (5) }
\end{aligned}
$$

Teniendo en cuenta que el ahorro de gobierno es igual a sus ingresos menos sus egresos, donde $Y g$ son los ingresos de gobierno y $G$ su gasto. $S g=Y g-G(6)$

Reemplazando 6 en 5 se obtiene que:

$$
\begin{gathered}
Y=C p+S p+C g+(Y g-G) \\
G=C g \\
Y=C p+S p+C g+(Y g-C g)(7)
\end{gathered}
$$

Se anula de la ecuación 7 el consumo de gobierno (Cg), de modo que: $Y=C p+S p+T(8)$

Despejando el ahorro privado $\mathrm{Sp}$, se evidencia que:

$$
S p=Y-T-C p(9)
$$

El ahorro privado entonces es igual al ingreso disponible $(Y-T)$ menos el consumo privado. En consecuencia, generalizando la identidad $S=1$ y desagregando el ahorro (S) entre ahorro privado (Sp), ahorro público (Sg) y ahorro externo (Se), se consigue la siguiente ecuación:

$$
\begin{gathered}
S=I=S p+S g+S e(10) \\
S e=X-M(11)
\end{gathered}
$$

Considerando que el ahorro externo es igual a la diferencia entre exportaciones e importaciones, se procede a reemplazar la ecuación 11 en 10 y se consigue establecer las tres brechas o equilibrios macroeconómicos que determinan el nivel de inversión en la economía.

$$
I=S p+S g+(X-M)
$$

\section{$I=(Y-C p-T)+(T-G)+(X-M)(12)$}

La ecuación número 12, además de evidenciar como se compone la inversión en una economía, permite evidenciar las variables en base a las cuales se constituirán las brechas de cada sector.

Para la construcción de las brechas en el caso ecuatoriano se consideró en la brecha pública ingresos y egresos del sector público no financiero (SPNF) y resto del SPNF, para la brecha externa (Exportaciones, importaciones, servicios, renta, transferencias corrientes, transferencias de capital y títulos de deuda), para la brecha privada (ingreso disponible, consumo e inversión privados).

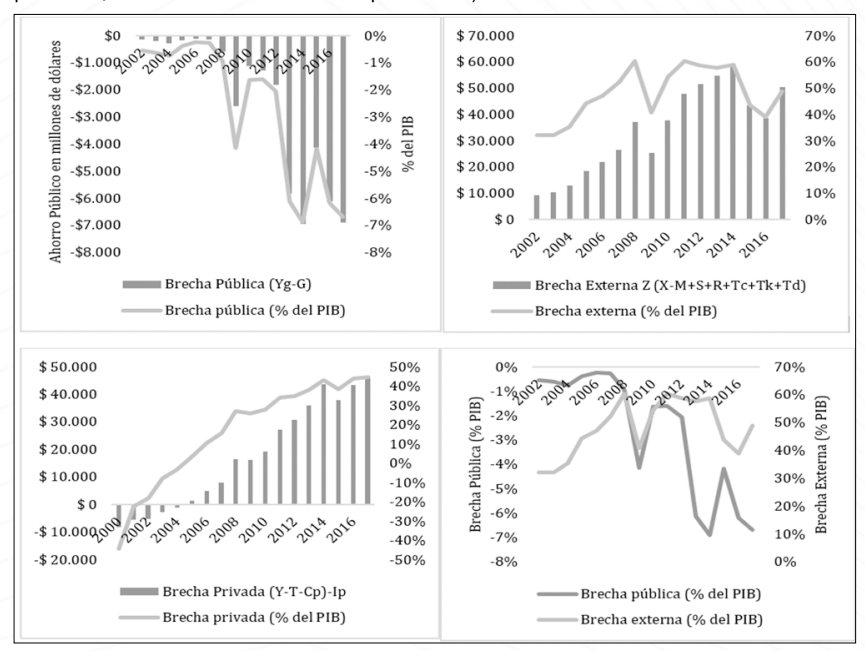

Fuente: Banco Central del Ecuador (BCE), Corporación Andina de Fomento (CAF) Figura 1. Brechas macroeconómicas del Ecuador en precios constantes de 2010

Al evidenciar gráficamente la evolución de las tres brechas a lo largo de los dos periodos de análisis (2000-06 y 2007-17) se evidencia como la brecha del sector público en el primer periodo refleja cifras positivas, es decir, los ingresos de gobierno fueron superiores a los egresos y representaban al 2006 un -0,2\% del PIB, sin embargo a partir de 2007 cuando el manejo de la política económica toma un giro drástico, la brecha fiscal comienza a reflejar cifras negativas (déficit fiscal $G>Y g$ ) y crecientes debido a los altos niveles de desahorro público, pasando de un $-4,1 \%$ en 2007 a -6,7\% en 2017 como porcentaje del PIB.
Si se analiza la brecha externa y la brecha fiscal en conjunto se puede evidenciar que su participación en el PIB fluctúa de manera bastante similar a partir del segundo periodo debido a la dependencia que tiene la brecha fiscal de la brecha externa determinada por factores exógenos.

Se evidencia como a raíz de una baja en los precios del crudo en 2009 , la brecha externa cae simultáneamente con el déficit fiscal y juntas vuelven a recuperarse con el alza del precio del crudo hasta 2014, cuando la economía vuelve a enfrentar un shock negativo por la baja en el crudo que afectó a la brecha externa y consecuentemente agravó el déficit fiscal.

Por otra parte, se puede observar que la brecha del sector privado refleja una tendencia creciente y sostenida durante ambos periodos, a pesar de que se ve ligeramente afectada en 2014 por el shock negativo externo de la baja en el precio del petróleo, este sector no evidencia una relación tan arraigada hacia shocks en la brecha externa como si lo refleja el sector público.

En base a la información expuesta anteriormente, puede sostenerse que en la economía ecuatoriana las brechas dominantes son la fiscal y la externa, presentando en la segunda una restricción derivada de la dependencia de la economía al precio del crudo que se determina de manera exógena por la demanda mundial.

En consecuencia, el análisis se enfoca en hacer evidente la relación entre la brecha fiscal y la externa, para lo cual, se ejemplificarán ambas en términos de cuentas nacionales, de modo que, la brecha fiscal es el déficit o superávit del sector público y, la brecha externa es la cuenta corriente de la balanza de pagos incluidos títulos de deuda y transferencias de capital.

En este sentido, basándose en Bacha (1990), se establece una condición en base a componentes de ambas brechas que determinan empíricamente la orientación del desarrollo de la productividad en una economía.

Los componentes utilizados para constituir esta condición son 'm' que se define como el contenido de bienes de capital importables en la inversión, de modo que, en términos de cuentas nacionales $m$ es la proporción que ocupan los bienes de capital en el total de importaciones, esto hace referencia a las importaciones que aportan al fomento de la productividad en la economía. Por otro lado, $k^{\star}$ refleja la participación de la inversión pública en la inversión total, es decir, la productividad que el gasto e gobierno brinda a la economía.

$$
\begin{aligned}
& \frac{1}{m}>1+\boldsymbol{k}^{*} ; \text { Motor de la economía: Estado } \\
& \frac{1}{m}<1+\boldsymbol{k}^{*} ; \text { Motor de la economía: S. Privado }
\end{aligned}
$$

En el primer caso, la productividad aportada por los bienes de capital importados es menor a la que aporta la inversión pública, de modo que, si esa condición se cumple, el motor de la economía y encargado de brindar productividad a la estructura productiva es el Estado.

Por otro lado, en el segundo caso la productividad aportada por la importación de bienes de capital (transferencia de tecnología) es mayor a la que aporta la inversión pública, en consecuencia, bajo esa condición el motor de la economía es el sector privado.

En base a las preposiciones mencionadas, se procede a calcular las condiciones en ambos periodos de análisis para el Ecuador. Los resultados en base a las condiciones expuestas resaltan de manera evidente el elemento diferenciador (desahorro público) de ambos periodos de análisis. 
Tabla 1. Determinación del sector motor de la economía

\begin{tabular}{cccc}
\hline Año & $(\mathbf{1} / \mathbf{m})-\left(\mathbf{1 + \mathbf { k } ^ { * } )}\right.$ & Motor & Vía \\
\hline $\mathbf{2 0 0 0}$ & $-5,2064$ & S. Privado & Transferencia de tecnología \\
$\mathbf{2 0 0 1}$ & $-1,8945$ & S. Privado & Transferencia de tecnología \\
$\mathbf{2 0 0 2}$ & $-3,8054$ & S. Privado & Transferencia de tecnología \\
$\mathbf{2 0 0 3}$ & $-2,8516$ & S. Privado & Transferencia de tecnología \\
$\mathbf{2 0 0 4}$ & $-2,6145$ & S. Privado & Transferencia de tecnología \\
$\mathbf{2 0 0 5}$ & $-2,9096$ & S. Privado & Transferencia de tecnología \\
$\mathbf{2 0 0 6}$ & $-2,3625$ & S. Privado & Transferencia de tecnología \\
$\mathbf{2 0 0 7}$ & $-0,3566$ & S. Privado & Transferencia de tecnología \\
$\mathbf{2 0 0 8}$ & 1,5804 & Estado & Inversión Pública \\
$\mathbf{2 0 0 9}$ & 1,1704 & Estado & Inversión Pública \\
$\mathbf{2 0 1 0}$ & 1,5455 & Estado & Inversión Pública \\
$\mathbf{2 0 1 1}$ & 1,9295 & Estado & Inversión Pública \\
$\mathbf{2 0 1 2}$ & 2,1850 & Estado & Inversión Pública \\
$\mathbf{2 0 1 3}$ & 2,2361 & Estado & Inversión Pública \\
$\mathbf{2 0 1 4}$ & 2,3796 & Estado & Inversión Pública \\
$\mathbf{2 0 1 5}$ & 2,0228 & Estado & Inversión Pública \\
$\mathbf{2 0 1 6}$ & 2,2721 & Estado & Inversión Pública \\
$\mathbf{2 0 1 7}$ & 2,2271 & Estado & Inversión Pública \\
\hline
\end{tabular}

Fuente: Banco Central del Ecuador (BCE), Corporación Andina de Fomento (CAF)

De 2000 a 2006, se evidencia una relación negativa, esto implica que la productividad generada por el volumen de importaciones de bienes de capital superó a la generada por la inversión pública catalogando a la economía en una orientación más privada.

Por otro lado, de 2008 a 2017 se obtiene una relación positiva, traduciéndose en que la inversión pública superó a la importación de bienes de capital al momento de generar productividad para la economía, de manera que, durante este segundo periodo de análisis se constituyó una 'State led economy'.

En este sentido, podría considerarse al signo negativo en 2007 como un breve periodo de transición de una economía privadamente orientada a una 'State led economy', dinámica explicada por el cambio en el manejo de la política económica. Esta preposición puede fortalecerse al comparar la evolución de la inversión pública y privada en términos reales a lo largo de ambos periodos de análisis.

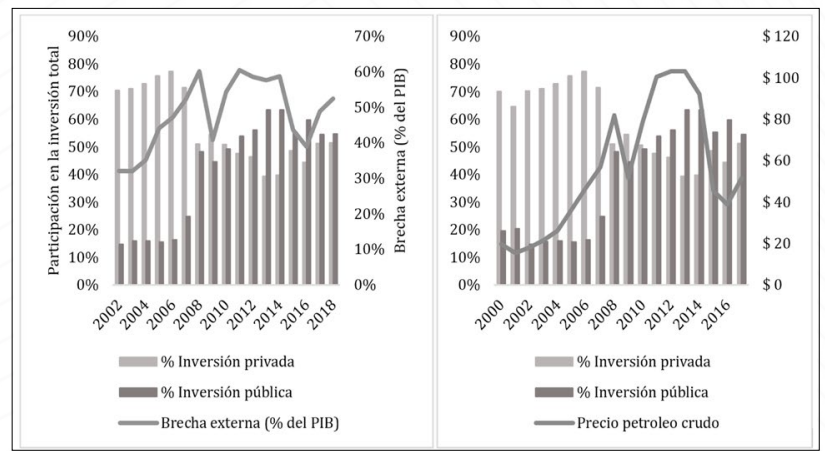

Fuente: Banco Central del Ecuador (BCE), Corporación Andina de Fomento (CAF Figura 2. Evolución de la inversión pública vs privada en la inversión total y sus determinantes

Como se puede evidenciar, durante el primer periodo de análisis se aprecia la imporante participación de la inversión privada en la economía en términos reales manteniendose en promedio por sobre el $70 \%$ del total de la inversión efectiva.

Por otra parte, en cuanto al segundo periodo de análisis se evidencia como la participación de la inversión pública va tomando fuerza desde 2007 superando el $20 \%$ en la participación de la inversión y posicionándose en sus mejores momentos por sobre el 60\% de la inversión total en 2014.

No obstante, a raíz del shock negativo externo ocasionado por la caída en el precio del barril de crudo que presionó a la baja a la brecha externa, se evidencia como la inversión pública cae por debajo de sus niveles máximos (60\%) confirmando la vulnerabilidad del sector público ante variaciones en la brecha externa.

Además, se evidencia como a pesar de la caída en el precio del crudo, la inversión pública no cae abruptamente en la misma magnitud, mas bien se mantiene sobre el $50 \%$ de la inversión total. Pero ¿cómo puede esto explicarse?
Si bien es cierto, el precio del petroleo se relaciona de manera directa con la evolución de la brecha externa mediante la balanza de bienes, no obstante, la brecha externa también se ve influenciada por otras cuentas de la balanza de pagos. En consecuencia, el diferencial entre la brecha externa y el precio del petroleo crudo es influenciado por esos otros factores entre los que se encuentran los títulos de deuda pública, rubro que tomó relevancia en el segundo periodo de análisis como se evidencia en el crecimiento real del stock de deuda pública.

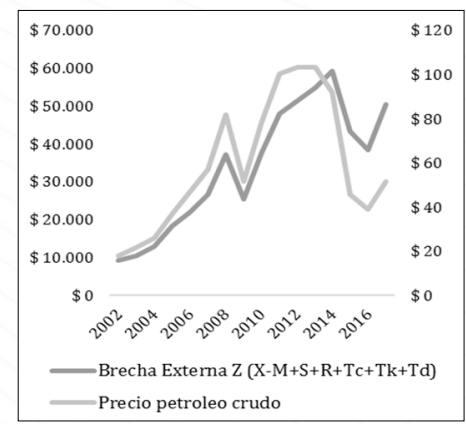

Fuente: Banco Central del Ecuador (BCE), Corporación Andina de Fomento (CAF)
Figura 3. Evolución brecha externa vs precio del petroleo

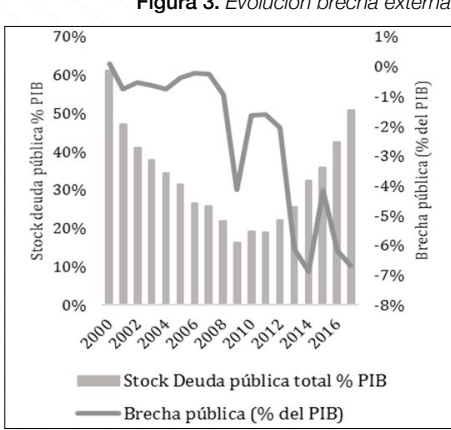

Fuente: Banco Central del Ecuador (BCE), Corporación Andina de Fomento (CAF)
Figura 4. Evolución real stock de deuda pública vs déficit fiscal

En este sentido, puede sostenerse que el diferencial entre la brecha externa y los precios del crudo tiene relación con el crecimiento del stock de deuda pública que ha venido auxiliando a mantener la inversión pública en niveles superiores al 50\%. No obstante, esto ha ocasionado que el stock real de la deuda pública supere el techo de la deuda del $40 \%$ del PIB establecido en el Código Orgánico de Planificación y Finanzas Públicas.

La cuestión principal está en que, independientemente de que sector sea el motor de la economía, el crecimiento real del producto (PIB) debe crecer sostenidamente y ser superior a la tasa de interés real por de la deuda pública, caso contrario la deuda puede volverse insostenible. Dicho de otro modo, el alza en la productividad de la economía debe superar al costo de la deuda.

En consecuencia, vale la pena introducir al análisis un modelo de sostenibilidad de la deuda pública considerando que el Estado tiene una vida infinita y que debido a que es pública, la pagará la sociedad con sus tributos, de manera que se medirá el cambio en el volumen de la deuda por unidad de producto. En este sentido, se procede a enunciar las variables necesarias, entre estas: el nivel de deuda pública existente al final del periodo anterior, Bt-1, el déficit primario, Xt, la tasa de interés real de la deuda, r, y el crecimiento real del producto, y. (Argandoña, 2006)

$$
\Delta B t=B_{t-1}+X_{t} \frac{(r-y)}{(1+r)}
$$

Al analizar la ecuación del crecimiento del volumen de deuda por unidad de producto se puede sostener que, el volumen de deuda pública aumenta cuando se produce un déficit primario por unidad de producto, $\mathrm{Xt}$, y también aumenta cuando el tipo de interés real, $r$, es mayor que la tasa de crecimiento del producto real, y. Adicionalmente, si r>y, y existe deuda pública vigente, la emisión de deuda adicional para cubrir los intereses de esa deuda derivarán en un aumento de la relación deuda PIB agravando el problema de la sostenibilidad. (Argandoña, 2006) 


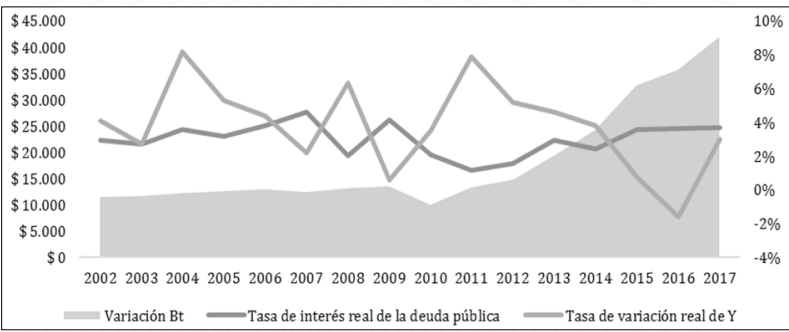

Fuente: Banco Central del Ecuador (BCE), Corporación Andina de Fomento (CAF), Ministerio de Economía y Finanzas (MEF) Figura 5. Evolución del cambio en la deuda pública por unidad de producto-Tasa interés real vs variación PIB real

Este gráfico representa la evolución de la variación del PIB real y de la tasa de interés real de la deuda pública, en este sentido, pueden evidenciarse dos periodos en los que la deuda se tornaba sostenible (2003-2007 y 2010-2014) y un periodo prolongado en el que la contratación de deuda se torna abruptamente insostenible (2015-2017).

Se evidencia además que, el volumen de deuda pública por unidad de producto mengua en 2009 debido al 'default' de la deuda ${ }^{6}$ que le permitió al Estado ecuatoriano reducir el stock de deuda, no obstante, seguido de esto se evidencia una evolución creciente y sostenida que fluctúa en relación inversa con la evolución de la brecha pública (déficit fiscal).

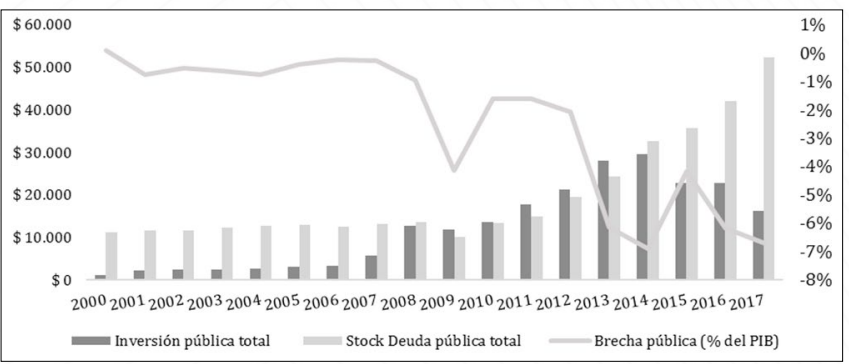

Fuente: Banco Central del Ecuador (BCE), Corporación Andina de Fomento (CAF), Ministerio Figura 6. Comparación stock de deuda pública vs inversión pública y evolución y real déficit fiscal

A pesar de que el volumen de deuda crece para canalizar la brecha fiscal amortiguando el shock externo en la economía, se evidencia que, si bien la inversión pública se mantiene por encima del 50\% de la inversión total, en términos nominales no se refleja un nivel constante sino más bien decreciente a partir de 2014.

Dicho de otra manera, a medida que ha aumentado el stock de deuda, la inversión pública no ha fluctuado en la misma dirección, esta dinámica agrava el problema de la sostenibilidad ya que, en una economía en la que el Estado es el motor que brinda la productividad a través de inversión pública, el hecho de que esta última se reduzca a la vez que aumentan las obligaciones a futuro, condena a la economía a un círculo vicioso de baja productividad y endeudamiento.

En consecuencia, considerando la vulnerabilidad del país ante shocks externos debido a la incapacidad de ejercer política monetaria por ser una economía dolarizada, las estrategias monetarias para brindar alivio a la condición mencionada en el párrafo anterior quedan descartadas. De manera que, soluciones a mediano y largo plazo recaen en estrategias que apunten a fortalecer la productividad en sectores exportadores y, estrategias a corto plazo recaen en programas de austeridad para el sector público.

\section{Conclusiones}

En base a todo lo expuesto anteriormente, puede sostenerse que la economía ecuatoriana enfrenta una restricción en la brecha o equilibrio externo derivado de la dependencia al precio del barril de crudo que es determinado de manera exógena por la demanda mundial.
Adicional a esto, se evidencia la relación existente entre la brecha externa y la brecha fiscal a partir del segundo periodo de análisis (2007-17) en donde, el Estado se posiciona como motor de la economía debido al aumento de su participación en la economía derivada del aumento de sus ingresos petroleros determinados por factores exógenos. En este sentido se puede argumentar que, las fluctuaciones en la demanda mundial que afecten a la baja al precio del barril de crudo vulnerarían las finanzas públicas, presionarían al déficit fiscal y afectarían a la actividad productiva de la economía estatal-petrolera en todos los sectores donde se filtren tales recursos.

Como respuesta, la estrategia contemplada a corto plazo ante choques petroleros externos ha recaído en la emisión de deuda con el fin de canalizar el desequilibrio en la brecha fiscal y generar ajuste con crecimiento en la 'State led economy'. No obstante, a pesar del crecimiento del stock de deuda, la inversión pública se ha reducido y la brecha fiscal se estima seguirá reflejando déficits constantes. De manera que, esta dinámica agravará la sostenibilidad de las finanzas públicas si los niveles de crecimiento del producto continúan siendo menores a las tasas de interés a las que el Estado contrate sus créditos.

De tal modo que, el Estado se encuentra en un contexto en el que no tiene otra alternativa que no sea reducir gradualmente su participación y presionar a la transición hacia una economía liderada por el sector privado, ya que los recursos estatales, tanto petroleros como tributarios, deberían tener la prioridad de ser destinados a cubrir las obligaciones crediticias existentes y futuras en función de los objetivos de austeridad y equilibrio, por lo que, ya no tendrán la capacidad de ejercer el rol de dinamizar la economía al ritmo que lo venían haciendo desde 2008 por medio del componente del gasto de gobierno.

En consecuencia, la transición mencionada en el párrafo anterior se presenta como un reto para el Ecuador, debido a que, en una economía en la que el Estado es el motor que brinda la productividad a través del gasto público, el hecho de que esta última se reduzca a la vez que aumentan las obligaciones (deuda) a futuro, condena a la economía a un círculo vicioso de baja productividad y endeudamiento que degradará el contexto económico si la contraparte privada no dinamiza de manera determinante la actividad productiva.

\section{Referencias}

Argandoña, A., \& Carrasquero, C. (2006). Análisis de la sostenibilidad de la deuda pública. Estrategia Financiera No232.

Bacha, E. (1990). A three-gap model of foreing trasnfers and the GDP growth rate in developing countries. Journal of Development Economics, 279-296. Bacha, E. L. (1984). Growth with limited supplies of foreign exchange: A reappraisal of the two-gap model. Economic structure and performance, 263-280. Chenery, \& Bruno. (1962). Development alteratives in a open economy. Economic Journal 57, 79-103.

Chenery, Hllis, \& Strout. (1966). Foreing assistance and economic development. American Economics Review 56, 679-733.

Christiansen, L., Prati, A., Ricci, L. A., \& Tressel, T. (2009). External Balance in Low-income counties. NBER, 265-322.

Edwards, S. (1995). Why are saving rates so different across countries? Cambridge Working Paper 5097.

Eyzaguirre, N. (1989). Ahorro e inversión bajo restricción externa y fiscal. El caso de Chile 1982-87. Santiago: ECLA.

Ghosh, A. R., \& Ostry, J. D. (1994). Export Instability and the External Balance in Developing Countries. Staff paper IMF 41, 214-235.

León, P., Celi, E., \& Carvajal, F. (s.f.). El modelo de tres brechas una perspectiva empirica para el caso ecuatoriano 1965-1993.

McKinnon, R. I. (1964). Foreign exchange constraints in economic development and efficient aid allocation. The Economic Journal 74, 388-409.

Romaguera, P., \& Contreras, D. (1992). Restricciones al crecimiento: aplicación de un modelo de brechas a la economía chilena. Colección Estudios CIEPLAN, 145-182. 